\title{
Impacts, adaptation et vulnérabilité des systèmes naturels et humains en Europe
}

\author{
Eric Martin'1, David Salas y Mélia 1 , Vincent Badeau², Christine Delire', \\ Jean-Pierre Gattuso ${ }^{3}$, Aude Lemonsu', Valéry Masson', \\ Grégoire Pigeon ${ }^{4}$, Mathieu Regimbeau ${ }^{4}$, Vincent Viguié 5 \\ 1 Centre national de recherches météorologiques - Groupe d'étude \\ de l'atmosphère météorologique, Météo-France / CNRS, Toulouse \\ 2 Écologie et écophysiologie forestières, \\ Institut national de la recherche agronomique, Nancy \\ 3 Laboratoire d'océanographie de Villefranche-sur-Mer, \\ Université Pierre-et-Marie-Curie / CNRS, Villefranche-sur-Mer \\ 4 Division Agrométéorologie, Météo-France, Toulouse \\ 5 Centre international de recherche sur l'environnement et le développement, \\ École des Ponts ParisTech, Nogent-sur-Marne
}

\section{Résumé}

Cet article analyse les impacts observés et projetés du changement climatique sur les systèmes humains et naturels, leur vulnérabilité et les possibilités d'adaptation. Il donne un aperçu des principaux résultats pour l'hydrologie, l'agriculture, les écosystèmes naturels, les infrastructures, le transport, l'énergie, le tourisme, la santé et les aspects sociaux. L'article reprend les principales conclusions du chapitre Europe de la contribution du Groupe II au 5e rapport du Giec paru en 2014. Plusieurs études traitant spécifiquement de la France métropolitaine sont également présentées, mais sans prétendre à l'exhaustivité, le sujet étant particulièrement vaste.

\section{Abstract}

Impact, adaptation and vulnerability of natural and human systems in Europe

This article analyses the observed and projected impacts of climate change on human and natural systems, their vulnerability and adaptation options. It provides insight into the main results related to hydrology, agriculture, natural ecosystems, transport, energy, tourism, infrastructures, health and social aspects. This article presents the main results concerning Europe that were compiled in the contribution of Working Group II to the IPCC fifth assessment report published in 2014 . Several studies focused on mainland France are also presented, without claiming to be exhaustive.

eric.martin@meteo.fr

e changement climatique en cours se traduit notamment par le réchauffement de l'atmosphère, des océans, la fonte des glaces, un changement des précipitations, une hausse du niveau marin et des modifications du cycle du carbone. Certains de ces changements sont d'ores et déjà attribuables aux activités humaines (Planton et al., 2015, ce numéro). Le réchauffement à venir dépendra des émissions de gaz à effet de serre dans le futur, mais aussi de la sensibilité climatique (Boucher et al., 2015, ce numéro). Ainsi, le scénario le plus optimiste, le RCP2.61 (qui implique des émissions pratiquement nulles voire négatives pour la fin du $\mathrm{XXI}^{\mathrm{e}}$ siècle) conduirait à un réchauffement supplémentaire de la température moyenne globale de l'ordre de $1{ }^{\circ} \mathrm{C}$ d'ici à 2100 .

Ce réchauffement serait proche de $4{ }^{\circ} \mathrm{C}$ pour le scénario le plus pessimiste (RCP8.5). La Terre a déjà connu des changements de températures de cet ordre : au dernier âge glaciaire, la température moyenne était environ $5{ }^{\circ} \mathrm{C}$ plus basse qu'aujourd'hui. La planète s'est réchauffée à un rythme très rapide suite à des processus naturels (jusqu'à $1,5^{\circ} \mathrm{C}$ en 1000 ans, voir MassonDelmotte et al., 2015, ce numéro), mais cela reste 10 fois plus lent que le réchauffement projeté d'ici la fin de ce siècle. Or, des études montrent que de nombreux écosystèmes ont été affectés et que beaucoup d'espèces végétales et animales se sont éteintes au cours des derniers millions d'années en dépit de variations climatiques plus lentes que celles observées aujourd'hui. Cela pose par exemple la question du devenir de la biodiversité au cours du prochain siècle. Les évolutions climatiques actuelles et à venir représentent également un défi majeur en raison de leur impact sur pratiquement toutes les activités humaines.

Le groupe II du Giec travaille sur les impacts, l'adaptation et la vulnérabilité. Il analyse le risque lié au changement climatique, pour différents secteurs et systèmes, tout en intégrant les éléments de contextes non liés au climat. Son rapport est composé de deux parties : la première traite des aspects globaux et sectoriels ; la deuxième aborde les problèmes sous l'angle régional. Cet article propose de faire le point sur les principales connaissances, les incertitudes relatives à ces impacts et les possibilités d'adaptation. Il présente des résultats d'études récentes menées sur la France ainsi que des éléments tirés du chapitre « Europe » du rapport (Kovats et al., 2014).

1. Profils représentatifs de concentrations (Representative Concentration Pathways). Plus le chiffre du RCP est grand, plus le scénario est "fort» en termes d'émissions de gaz à effet de serre. Les chiffres vont de 2.6 (" très optimiste " a 8.5 «très pessimiste »). Voir Boucher et al. (2015, ce numéro) pour des descriptions plus complètes de ces scénarios et Guivarch et Cassen (2015, ce numéro) pour plus de détails sur les émissions globales de $\mathrm{CO}_{2}$ compatibles avec les différents scénarios. 


\section{Adaptation : quels sont les enjeux et les options possibles?}

En quoi le passage à de nouveaux régimes climatiques est-il préoccupant?

Mis à part certains cas extrêmes (climats désertiques, par exemple), il est difficile de dire que certains climats sont mieux adaptés que d'autres à l'Homme. En revanche, la densité de la population, les modes de vie, les infrastructures, les techniques agricoles y sont fort différents car adaptés aux climats locaux : c'est lorsque, pour une raison ou pour une autre, une société n'est pas bien adaptée à son climat qu'elle commence à être vulnérable. La canicule de 2003 en est une très bonne illustration : ses dégâts furent extrêmement importants à Paris, alors qu'il s'agissait de températures couramment observées l'été dans le sud de l'Espagne, où elles ne provoquent pas de conséquences aussi tragiques.

Le véritable enjeu du changement climatique est ainsi de savoir comment la transition peut être gérée, autrement dit comment passer de territoires adaptés à notre climat actuel à des territoires adaptés au climat futur et ce, en quelques décennies. Au-delà des enjeux techniques, un certain nombre de problèmes généraux se posent, qui doivent être pris en compte et rendent l'analyse extrêmement ardue. Ces problèmes sont liés au fait que, face à tout changement, le grand défi est la gestion de l'inertie. La société est vulnérable face à un changement si elle n'arrive pas à changer au même rythme ou à anticiper. Or, faire face au changement climatique suppose de modifier des infrastructures ou des modes d'organisation locale (spécialisation économique d'une région, par exemple) qui ne peuvent pas changer du jour au lendemain. Il faut donc prendre le problème le plus en amont possible.

Deux écueils principaux rendent l'adaptation particulièrement difficile. Tout d'abord, le climat évolue progressivement. L'objectif n'est ainsi pas d'être parfaitement adapté au climat de 2050 ou de 2100, mais d'être adapté à un climat naturellement variable, qui évoluera progressivement sous l'action de l'Homme. Le deuxième écueil est l'incertitude sur le climat futur, qui fait que même si l'on sait qu'il faut anticiper, on ne sait pas très bien à quoi se préparer. Cette incertitude a deux causes bien distinctes : d'une part notre connaissance imparfaite des mécanismes climatiques et d'autre part l'impossibilité de prévoir les émissions futures.

Quels choix faire et quand?

Par exemple, quelle décision prendre sur tel investissement qui va porter sur plusieurs dizaines d'années ? Une solution est de privilégier le principe de précaution : choisir de s'adapter systématiquement au pire des cas possibles. Ce choix a un sens, mais il est difficilement généralisable en pratique, car il porte en lui le risque de payer trop cher pour un résultat qui n'en valait pas la peine. Un certain nombre d'autres solutions existent, qui permettent de s'adapter au changement tout en réduisant au maximum le risque de coûts exagérés (Hallegatte, 2009).

Un premier type d'actions possibles vient de l'idée que nous sommes déjà aujourd'hui vulnérables à un certain nombre de problèmes environnementaux : la biodiversité est menacée, l'eau est fréquemment consommée de manière non durable une fraction non négligeable de la population vit en zone inondable, etc. Une part importante des conséquences projetées du changement climatique correspond à une amplification de ces problèmes déjà existants : par exemple, la ressource en eau risque de décroître là où aujourd'hui elle est souvent déjà mal gérée, ou encore la fréquence des inondations va augmenter dans un certain nombre de zones déjà inondables à l'heure actuelle. Une mesure d'adaptation simple, dans ce cas, consiste simplement à commencer par mieux gérer la situation actuelle. C'est ce que I'on appelle une mesure " sans-regret », c'est-à-dire qu'elle est bénéfique dans tous les cas, à court terme aussi bien qu'à long terme, et ce, quelle que soit l'ampleur future du changement climatique. Ce type de mesures n'est pas simple à mettre en place (elles l'auraient déjà été, sinon) et ne permet pas de s'adapter à l'ensemble des impacts climatiques. Mais elles constituent une première étape efficace.

Un autre type de mesures consiste à réduire la durée de vie des investissements et à tenter d'éviter, autant que possible, ceux de longue durée. Un investissement dont on espère une rentabilité sur 10 ans sera en effet plus évolutif et moins affecté par les diverses évolutions possibles du climat qu'un investissement dont on espère une rentabilité sur 20 ans ou plus.

Enfin, il existe presque toujours de nombreuses manières différentes de s'adapter au changement climatique, les situations avec une unique solution étant extrêmement rares. Il est alors préférable, autant que possible, de favoriser les stratégies, financières ou institutionnelles, d'adaptation réversibles et permettant de revenir en arrière à moindre coût, plutôt que les investissements dans des solutions technologiques coûteuses où l'argent, une fois investi, serait perdu. Par exemple, face à une augmentation du risque d'inondation, il est schématiquement possible de favoriser des plans d'occupation des sols restrictifs, interdisant d'aller s'établir dans les zones qui deviendront potentiellement inondables, ou bien de construire des ouvrages de défense (digues). Le grand inconvénient de la deuxième solution est que si l'on s'aperçoi quelques années après la construction des ouvrages que le risque a été surestimé, une quantité importante d'argent aura été dépensée en vain. À l'inverse, les interdictions émanan des plans d'occupation des sols peuvent être levées sans perte s'il s'avère qu'elles sont trop restrictives.

Toutes ces mesures ont bien entendu un coût à court terme et il n'est pas forcément simple de les mettre en œuvre. Réduire la durée de vie des investissements conduit à en choisir de moins ambitieux. Investir dans les marges de sécurité n'est pas gratuit et n'entraine pas de gain à court terme. Les stratégies réversibles ne sont pas les plus simples à mettre en œuvre et à faire respecter. Ce coût doit cependant être vu comme une assurance, que l'on paye initialement, mais qui limite les possibilités de pertes futures.

\section{Hydrologie et ressources en eau}

Les modifications du cycle de l'eau liées au changement climatique ont des conséquences importantes dans pratiquement tous les domaines. Elles interagissent directement ou indirectement avec des facteurs de changement non climatiques et avec les pratiques de gestion de l'eau.

\section{Quelques tendances climatiques observées à l'échelle du continent européen}

La synthèse effectuée par le Giec dans le chapitre " Europe » du rapport du groupe II nous indique que, depuis 1950, les précipitations ont augmenté dans le nord de l'Europe (jusqu'à +70 mm/an par décennie) et diminué dans certaines régions du sud de l'Europe (AEE, 2012). Concernant les débits des rivières, les tendances climatiques ne peuvent être estimées que pour des rivières peu influencées par l'action directe de l'Homme, en général des bassins-versants de dimension modeste. Une diminution des débits dans le sud et l'est de l'Europe a été notée depuis les années 1960. Les tendances positives observées sur les températures ont également pu avoir une 
influence sur ces variations, sachant que, toutes choses égales par ailleurs, le besoin en eau des plantes (la demande évaporative) croît avec la température, et que pour des grands bassins, les quantités d'eau évaporées sont d'environ deux tiers des précipitations. Concernant les rivières ayant un régime nival ou glaciaire, c'est la saisonnalité des débits qui est affectée : pic printanier de fonte nivale avancé, augmentation du débit estival en raison de la fonte accélérée des glaciers.

Sur la même période, des tendances à l'augmentation des crues ont été observées dans certaines régions (nord-est de la France, Allemagne, Europe centrale), alors que d'autres études n'ont pas noté de tendance dans d'autres régions (Suisse, pays scandinaves). Même quand des tendances nettes sont observées, leur attribution au changement climatique provoqué par les activités humaines n'est pas formellement établie. En effet, la variabilité naturelle du climat peut dominer le signal anthropique. Certains événements, tels que les inondations en Grande-Bretagne en 2007 ou en Allemagne en 2013 ont suscité l'attention des médias et, d'une manière générale, une augmentation des dommages a été observée. Cependant, cette tendance résulte principalement d'une plus grande exposition des personnes et des biens dans des zones à risques.

\section{Les projections climatiques concernant le cycle de l'eau}

En matière de projections (Boucher et $a l ., 2015$, ce numéro) du cycle de l'eau sur l'Europe, les résultats du $5^{\mathrm{e}}$ rapport du Giec confirment les résultats du précédent en précisant spatialement les résultats. Ils permettent aussi une meilleure estimation des extrêmes. Pour ce qui concerne l'Europe, les scénarios climatiques ont été récemment affinés grâce à des simulations régionales réalisées dans le cadre du projet international Cordex ${ }^{2}$. Dans les simulations, le réchauffement attendu est maximal sur l'Europe du Nord $\left(2,9^{\circ} \mathrm{C}\right.$ pour le scénario RCP4.5 pour la période 2071-2100 par rapport à 1971-2000), alors que pour les autres régions il varie de 1,7 à $2,1^{\circ} \mathrm{C}$. Pour le scénario RCP8.5, les chiffres sont respectivement de 5,2 et 3,2 à $4,1{ }^{\circ} \mathrm{C}$. Le réchauffement est maximal en été sur la région méditerranéenne et en hiver sur l'Europe du Nord. Pour les précipitations, les modèles s'accordent sur une augmentation en Europe du Nord et une baisse en Europe

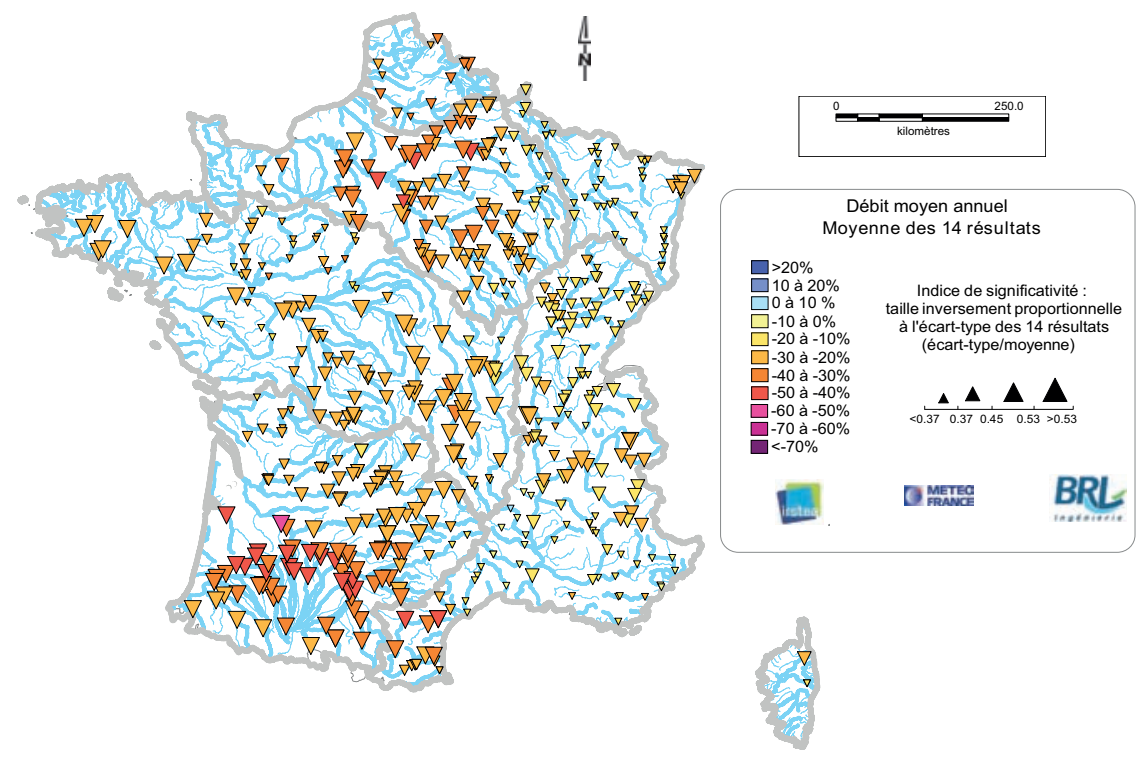

Figure 1. Évolutions relatives (en \%) du débit moyen annuel des rivières françaises entre 1961-1990 et 2046-2065. Moyenne des 14 simulations du projet Explore 2070. La couleur des triangles est fonction de l'intensité du changement. Leur taille indique la dispersion des simulations. Source : projet Explore 2070.

du Sud. Le phénomène le plus marquant pour les impacts est sans nul doute la forte augmentation attendue des extrêmes : vagues de chaleur, sécheresses et fortes précipitations. L'augmentation de ces phénomènes est d'autant plus forte que le scénario d'émission est fort. Il existe cependant des différences régionales ; ainsi, les fortes précipitations devraient augmenter surtout dans le nord de l'Europe, ainsi qu'en Europe centrale (sauf en été pour cette dernière). Les conséquences sur l'hydrologie seront logiquement extrêmement fortes. Les tendances déjà observées sur le continent européen vont se poursuivre ; ainsi, la fréquence et l'intensité des sécheresses (quelles que soient leurs définitions : météorologique, agricole ou hydrologique) augmenteront. Là encore, la zone méditerranéenne sera principalement touchée, mais des changements forts sont aussi à attendre en Europe centrale.

Sur la France, le projet Explore 2070 (Chauveau et al., 2013), piloté par le ministère de l'Écologie, du Développement durable et de l'Énergie, a étudié l'impact du changement climatique sur les débits des rivières dans l'hypothèse du scénario d'émissions médian $\mathrm{A}^{1} \mathrm{~B}^{3}$, à partir des résultats de sept modèles climatiques régionalisés par méthode statistique alimentant deux modèles hydrologiques. Pour une majorité de stations, en 2050, la baisse du débit moyen annuel serait de 10 à $40 \%$ par rapport à la période 1961-1990 (figure 1). Le bassin de la Garonne est particulièrement touché, avec une baisse allant jusqu'à $50 \%$, ainsi que le bassin de la
Seine, en raison d'une augmentation de l'évapotranspiration, combinée à une baisse relative des précipitations. Le sud-est de la France ne subit pas de fortes baisses en pourcentage, ce qui ne veut pas dire que l'impact réel soit faible, compte tenu de la rareté de la ressource en eau dans cette région. Les étiages devraient être plus sévères (figure 2). Les variations devraient être plus fortes que sur le débit moyen, mais ces chiffres sont à prendre avec prudence, compte tenu des difficultés des modèles à restituer les étiages dans les conditions actuelles. On peut noter que les débits d'étiage augmentent pour cerfait d'un accroissement significatif des débits des étiages hivernaux lié à la part croissante des précipitations sous forme pluvieuse (les précipitations ne sont plus stockées l'hiver sous forme de neige, entraînant donc un changement dans la saisonnalité des débits).

En ce qui concerne les crues, seules les crues journalières décennales ont été étudiées, et même si des variations de fréquence sont visibles (hausse dans les Cévennes et le nord-est de la France), les résultats sont entachés de fortes incertitudes. À la quantité d'eau, il faut aussi ajouter les problèmes de dégradation de la qualité de l'eau (plus difficile à maintenir en cas de sécheresse mais aussi d'inondation).

\footnotetext{
2. Coordinated Regional Climate Downscaling Experiment.

3. Il s'agit d'un scénario d'émissions de gaz à effet de serre appartenant à l'ancienne génération SRES (Special Report on Emissions Scenarios) de scénarios du Giec.
} taines stations des Alpes : il s'agit en 
En conclusion, il ne fait nul doute que les changements attendus auront des impacts marqués sur la plupart des secteurs socio-économiques, compte tenu des usages actuels de l'eau (irrigation, eau potable, hydroélectricité, besoin de maintenir un débit minimum pour garantir le bon état écologique des cours d'eau) et des tensions qu'ils exercent déjà sur la ressource dans de nombreuses régions. Il existe de nombreuses voies d'adaptation possibles, reposant par exemple sur l'augmentation du stockage d'eau ou la réduction de la demande des différents secteurs d'activité. Compte tenu du rôle central de la ressource en eau dans notre société, il sera nécessaire de se baser sur des études multisectorielles (qui sont malheureusement encore très peu nombreuses, Kovats et al., 2014) pour définir des politiques d'adaptation viables.

\section{Impacts sur l'agriculture}

L'agriculture est évidemment fortement dépendante des conditions climatiques. Cependant, elle dépend également d'autres facteurs qui peuvent varier à des échelles de temps comparables. Citons le cas de l'évolution des techniques agricoles avec la mécanisation, l'apport d'engrais, l'irrigation ou encore la sélection variétale pour les plantes et la sélection animale pour le bétail. Dans les études d'impact sur l'agriculture, estimer la seule contribution du climat aux variations de

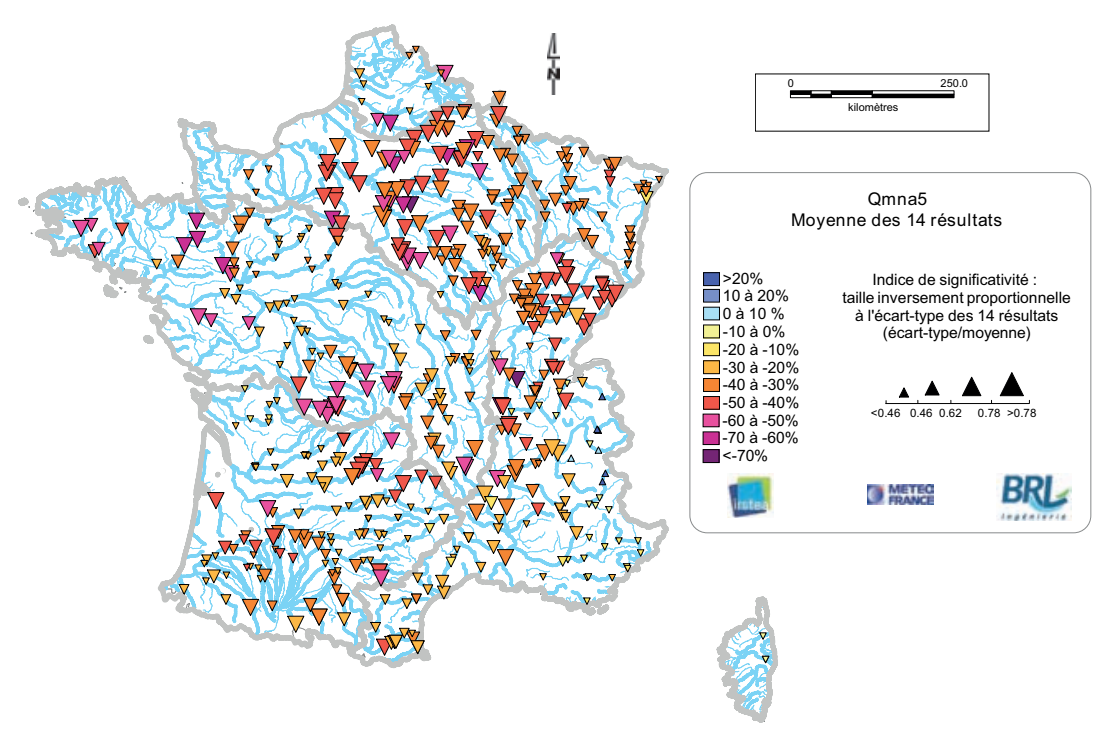

Figure 2. Évolution (en \%) du débit mensuel minimum quinquennal sec (le débit mensuel minimum se situe en dessous de ce seuil une année sur cinq, Qmna5) entre 1961-1990 et 2046-2065. Moyenne des 14 simulations du projet Explore 2070. La couleur des triangles est fonction de l'intensité du changement. Leur taille indique la dispersion des simulations. Source : projet Explore 2070. rendements agricoles ou aux évolutions des cycles de culture reste une difficulté majeure. Le recours à des modèles agronomiques, étalonnés à partir d'expériences de laboratoire ou de statistiques de rendements agricoles, est quasi systématique puisqu'il permet de tester un grand nombre d'hypothèses. Les résultats de ces modèles sont alors à exploiter en accord avec leurs hypothèses de départ.

Les paramètres climatiques qui influencent l'agriculture sont en premier lieu la température et les précipitations, mais aussi des phénomènes extrêmes tels que la grêle. Il faut également associer à ces paramètres les modifications de composition atmosphérique dont l'augmentation de la concentration en dioxyde de carbone $\left(\mathrm{CO}_{2}\right)$.

Dans le cas de la température et en considérant une bonne disponibilité en eau, la croissance des plantes tend à augmenter avec la température jusqu'à un certain seuil au-delà de laquelle elle diminue. Cette température seuil est appelée température d'échaudage et sa valeur est spécifique pour chacune des plantes (par exemple, de l'ordre de $28{ }^{\circ} \mathrm{C}$ pour le blé). Ainsi, schématiquement, l'augmentation des températures pendant la période hivernale ou de printemps tend à accélérer la croissance des cultures d'hiver comme le blé et avance les dates d'atteinte des stades de développement des plantes. En revanche, l'augmentation attendue de l'intensité et de la fréquence des épisodes caniculaires a des conséquences plutôt

négatives. Par ailleurs, certaines cultures (blé d'hiver ou fraises, par exemple, mais aussi certains arbres fruitiers) ont des besoins en froid qui contrôlent leurs périodes de dormance et de croissance annuelle. Pour ces cultures, l'augmentation des températures hivernales peut entraîner des dérèglements de leurs cycles.

Les précipitations conditionnent la satisfaction du besoin en eau des plantes. L'augmentation des températures contribue à une augmentation du besoin en eau des plantes. Le $\mathrm{CO}_{2}$ présent dans l'atmosphère est quant à lui absorbé par les plantes et transformé lors de la photosynthèse en différentes formes de réserve (fruits, tubercules, etc.) et d'autres éléments de structure de la plante (feuilles, tiges, etc.). L'augmentation de la concentration en $\mathrm{CO}_{2}$ a deux impacts sur le développement des cultures. Le premier est un effet fertilisant, c'est-àdire que la capacité de production des plantes s'en trouve augmentée. Cet effet est plus fort pour certaines catégories de plantes comme le blé, le riz ou l'herbe des prairies que pour d'autres catégories comme les plantes d'origine tropicale comme le maïs ou la canne à sucre. Le deuxième impact est un effet antitranspirant. La captation du $\mathrm{CO}_{2}$ se fait par des orifices sur les feuilles, les stomates, par lesquels les plantes transpirent. Une concentration accrue en $\mathrm{CO}_{2}$ dans l'atmosphère permet aux plantes de capter la même quantité de $\mathrm{CO}_{2}$ tout en limitant leur transpiration. Cet effet est valable pour l'ensemble des plantes. Ces augmentations concomitantes de la concentration en $\mathrm{CO}_{2}$ et de la température ont des effets antagonistes; les connaissances actuelles ne permettent pas de conclure si au final le besoin en eau des plantes va augmenter ou diminuer.

\section{Observations et projections pour les cultures}

Parmi les études menées sur l'évolution des cultures en relation avec l'évolution du climat, plusieurs constats ont été établis. Le premier concerne le décalage des cycles. Les analyses conduites pendant le programme Acta-Mires ${ }^{4}$ ont montré que le démarrage printanier

4. Acta : Association de coordination technique agricole. Mires : Mission interministérielle recherche et enseignement supérieur (Acta-Mires, 2009). 
de la croissance des prairies avait avancé de 2 à 16 jours au cours des 30 dernières années sur le territoire métropolitain. Cela permet de gagner en moyenne 5 jours de pâturage au printemps. De même, un allongement de la pousse est constaté à l'automne et permet de gagner une semaine de pâturage. Des décalages sont également observés pour d'autres cultures comme des avancées de date de vendanges pour la vigne. Même si le climat n'est pas le seul facteur contribuant à ces variations, il en explique une grande part. En se tournant vers le futur à l'aide des projections climatiques, des évolutions sont encore à attendre.

Un deuxième constat concerne les variations de rendements. En Europe, par exemple, Brisson et al. (2010) ont cherché à expliquer la stagnation des rendements de blé observée depuis le milieu des années 1990. Parmi les différents facteurs mis en cause, ils déterminent que le climat est le facteur dominant. À plus grande échelle, le groupe II du Giec a recensé un grand nombre d'études d'évolution des rendements à la fois sur la période historique (à partir de 1950) mais également sur le futur (figure 3). Pour la période historique, trois études sur quatre concluent à un impact négatif de l'évolution climatique sur les rendements. Pour le futur, la proportion d'études relatant une tendance à la baisse est similaire. La prise en compte de mesures d'adaptation permet de légèrement limiter cette tendance (Porter et al., 2014). Une étude sur quatre conclut à une tendance à la hausse des rendements dans des régions très spécifiques comme la Sibérie ou le nord de la Chine, pour lesquelles les températures sont aujourd'hui des conditions limitantes à la croissance des végétaux. Le dernier point important concerne les projections d'une augmentation de la variabilité interannuelle des rendements en réponse aux variations du climat. Une augmentation de la variabilité de la production a un impact négatif sur la sécurité alimentaire par le biais des variations qu'elle impose aux marchés.

Face à ces changements déjà en cours, des études d'adaptation sont menées à tous les niveaux. Par exemple, en France, le ministère de l'Agriculture, de l'Agroalimentaire et des Forêts a rassemblé autour de lui de nombreux acteurs pour conduire la prospective
Afclim (Agriculture, forêt, climat : vers des stratégies d'adaptation; Vert et al., 2013). À partir de cas d'études, des diagnostics ont été posés et des options d'adaptation identifiées. On peut citer par exemple, pour la culture du blé, une stratégie consistant à avancer le cycle de culture (avancée des semis, recherche de variétés plus précoces) pour esquiver les périodes potentiellement exposées au risque de sécheresse en fin de cycle. Cependant, ce type de stratégie n'est pas envisageable pour une culture d'été comme le maïs.

\section{Impact sur les forêts et leur vulnérabilité}

Le changement climatique affecte aussi les forêts, qu'elles soient naturelles ou gérées par 1'Homme, notamment en modifiant la croissance des arbres, en favorisant les dégâts causés par le feu, les sécheresses ou les tempêtes, ou en favorisant divers ravageurs (maladies et insectes). L'évolution à venir du climat devrait conduire à une modification de l'aire de répartition des différentes espèces d'arbres. (a) Impact du changement climatique sur les rendements moyens

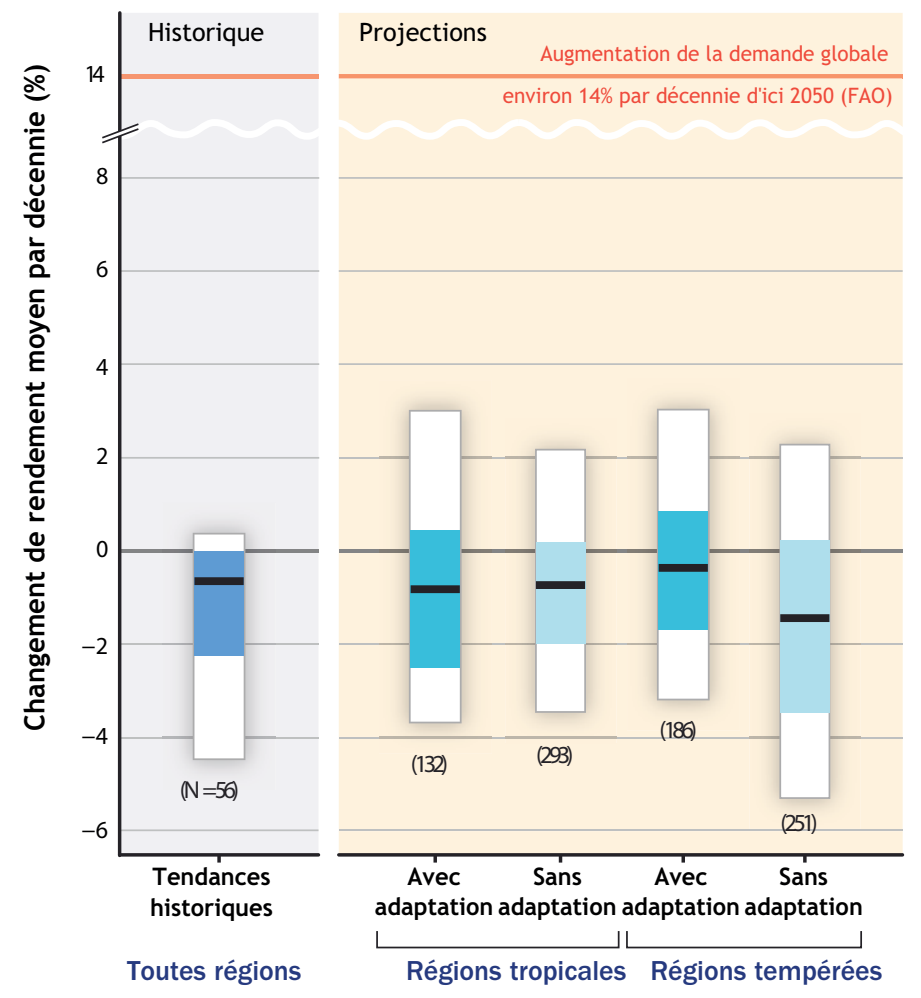

(b) Impact sur la variabilité interannuelle des rendements

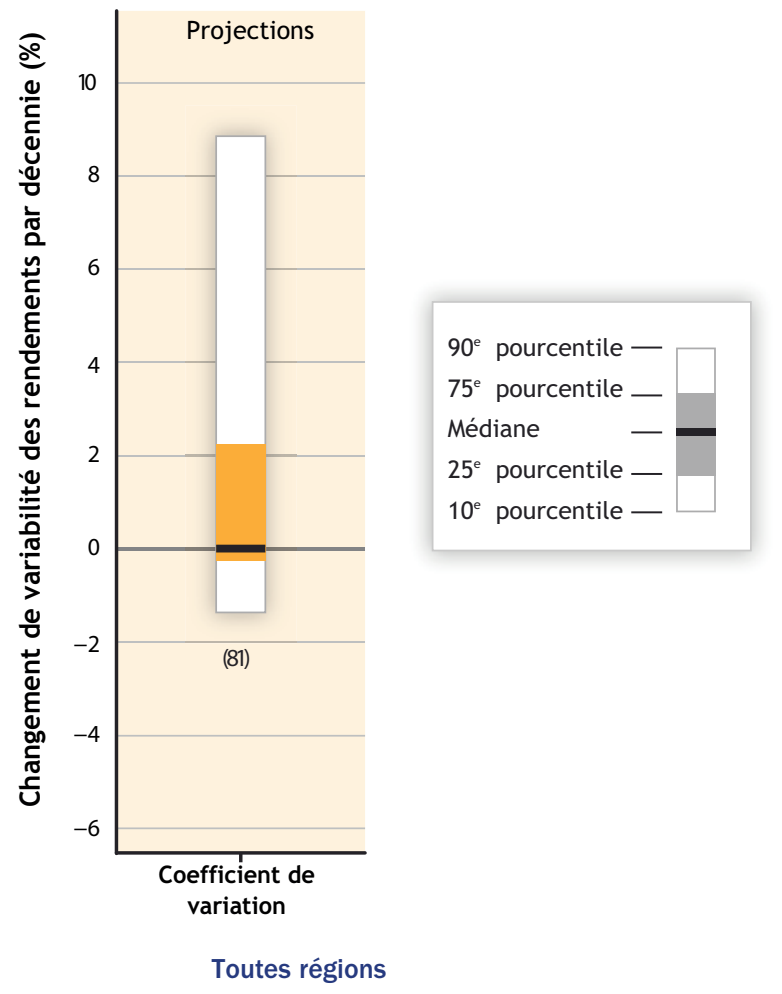

Figure 3. Quantification de l'impact des changements du climat et de la concentration en $\mathrm{CO}_{2}$ sur les rendements agricoles. Cette figure inclut les impacts historiques et projetés pour (a) les rendements moyens (respectivement partie gauche et droite) et (b) leur variabilité interannuelle. Les tendances sont indiquées en pourcentage de variation du rendement moyen par décennie. Pour chaque cas de l'axe horizontal est indiqué le nombre d'études exploitées. II est également fait mention de la tendance d'augmentation de la demande en produits agricoles. Source : figure 7.7 de Porter et al. (2014). 
Certaines régions vont devenir favorables à l'installation d'essences non présentes initialement ou au contraire inadaptées à d'autres essences présentes historiquement. Il est possible de quantifier ces évolutions en utilisant des modèles de végétation. Ainsi, dans le cas de la France, pour le $\mathrm{XX}^{\mathrm{e}}$ siècle, le modèle Ibis (Cheaib et al., 2012) simule des forêts dominées par des arbres à feuilles caduques (figure $4 \mathrm{a}$ ) et des conifères sur la plus grande partie du pays. Sur le pourtour méditerranéen, le modèle simule un mélange de forêts à feuilles persistantes et de maquis d'arbustes, ce qui correspond bien à la végétation naturelle en France. Pour la fin du XXI ${ }^{\mathrm{e}}$ siècle, selon le même modèle, les forêts de feuillus à feuilles caduques les plus productives seront reléguées dans le quart nord-est du pays (figure 4b), tandis que des forêts semblables aux forêts méditerranéennes actuelles prédomineront dans l'Ouest et le SudOuest.

\section{Rôle des extrêmes}

Certains événements extrêmes peuvent aussi directement affecter les forêts selon des échelles de temps bien plus courtes. Jusqu'à présent, plusieurs périodes de sécheresse intense ont entraîné une mortalité des arbres et un recul associé de certaines forêts, principalement dans le sud de l'Europe. Même si l'on ne peut pas attribuer formellement au changement climatique certaines tempêtes récentes particulièrement dévastatrices, telles que Klaus en janvier 2009, il a été observé que les dommages causés par les tempêtes sur les forêts européennes ont récemment augmenté. On estime également que, sous l'effet du réchauffement climatique, les tempêtes de printemps et d'automne devraient affecter plus sévèrement les forêts à la fin $\mathrm{du} \mathrm{XXI}{ }^{\mathrm{e}}$ siècle qu'actuellement, car les sols resteront gelés pendant une période plus courte, ce qui favorise le déracinement des arbres. Par ailleurs, le changement climatique devrait se traduire par des hivers plus doux et des sécheresses plus fréquentes. Il s'agit là de conditions favorables au développement et à la propagation de maladies des arbres liées à certains champignons microscopiques. Parallèlement, le réchauffement du climat pourrait permettre à des insectes tels que le bostryche typographe (coléoptère ravageant les forêts d'épicéa) d'étendre leur habitat à la Scandinavie et à certaines régions d'Europe centrale.

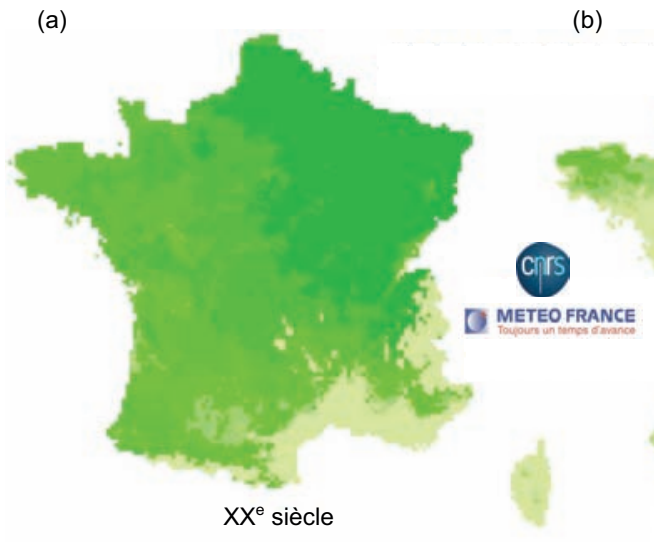

(b)

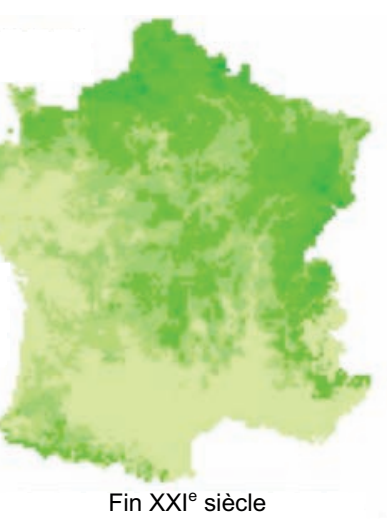

$\begin{array}{llllll}0.25 & 0.3 & 0.35 & 0.4 & 0.45 & 0.5\end{array}$

Figure 4. Productivité primaire des arbres à feuilles caduques simulée par le modèle lbis pour la végétation naturelle pour le XXe siècle (a) et la fin du XXIe siècle (b), pour le scénario à fortes émissions de gaz à effet de serre SRES-A2 du Giec.

\section{Évolution des zones sensibles aux feux}

Plusieurs études évaluées par le groupe II du Giec font état d'une augmentation de l'activité des incendies de forêt dans certaines régions du monde en termes de nombre, de durée et de surface brûlée. C'est le cas notamment pour les régions de latitude élevée couvertes de forêts de conifères et de toundra, en Amérique du Nord ou en Russie. En France, une mission interministérielle a été menée pour évaluer l'évolution des zones géographiques sensibles aux feux ${ }^{5}$. La méthodologie de cette étude reposait sur l'analyse de l'indice feu météo (IFM) qui est calculé selon les conditions météorologiques telles que la température, l'humidité relative, la vitesse du vent et la pluie. Plus la valeur de l'IFM est élevée, plus les conditions météorologiques sont propices aux incendies. Cependant, il n'est pas directement possible de relier une valeur donnée d'IFM à un niveau de risque global d'incendie puisque l'effet anthropique n'est pas pris en compte. On peut notamment trouver des situations où le risque global de feu est aggravé par rapport au risque météorologique ou au contraire réduit par l'influence du dispositif préventif, l'évolution des moyens de lutte contre les incendies, les facteurs déclencheurs d'incendies, etc. L'analyse de la période 1958-2008 a fait apparaître une augmentation significative de l'IFM moyen annuel. Cette hausse, de l'ordre de $20 \%$, s'est accompagnée d'un allongement de la saison de feu et d'une extension de la zone marquée par un risque très élevé. Enfin, selon différents scénarios de projection climatique, le risque météorologique d'incendie augmente de l'ordre de $30 \%$ à l'horizon 2040 par rapport à la période 1961-2000, avec des extrêmes plus marqués. À l'horizon 2060, les valeurs de l'IFM augmentent de $75 \%$ : l'année 2003 deviendrait alors la norme en termes de risque météorologique d'incendie. Cette évolution des indices IFM a été croisée avec l'évolution des massifs forestiers afin d'établir une cartographie des zones potentiellement sensibles aux feux de forêt comme présenté sur la figure 5. À l'horizon 2060, les zones potentiellement sensibles aux incendies de forêt s'étendent et des régions comme le massif des Landes ou la Sologne pourraient avoir une sensibilité égale à celle du bassin méditerranéen aujourd'hui.

\section{Impact sur les écosystèmes terrestres et d'eau douce 6}

On s'attend à ce que l'impact du changement climatique sur les écosystèmes terrestres et d'eau douce devienne majeur au cours de la seconde moitié du XXI ${ }^{\mathrm{e}}$ siècle, particulièrement dans le cas des scénarios à fortes émissions RCP6.0 et RCP8.5 (voir Boucher et al.,

5. Le rapport de la mission interministérielle "Changement climatique et extension des zones sensibles aux feux de forêts " a été livré en juillet 2010. Il est accessible à l'adresse suivante. $w w w$.ladocumentationfrancaise.fr/rapportspublics/104000494/index.shtml

6. La plupart des résultats présentés dans cette partie et les parties suivantes (à l'exception de celle portant sur les écosystèmes marins) sont directement issus du chapitre "Europe 》 du dernier rapport du groupe II du Giec (Kovats et al., 2014). 
2015, ce numéro). D'ici là, les principaux facteurs menaçant ces écosystèmes restent les changements d'occupation des sols, la pollution et le développement de la captation d'eau.

L'impact du changement climatique sur les espèces animales et végétales, regardé seulement sous l'angle du changement de température et d'une manière très générale, peut être évalué d'une manière relativement simple. Le principe est de comparer la vitesse à laquelle peuvent migrer ces espèces avec la " vitesse de déplacement » des climats. Par exemple, la température moyenne annuelle actuelle de Nantes est comparable à celle de Bordeaux il y a 100 ans (réchauffement de près de $1{ }^{\circ} \mathrm{C}$ ). Les deux villes étant distantes de près de $300 \mathrm{~km}$, on peut conclure que le climat s'est "déplacé » d'environ $30 \mathrm{~km}$ par décennie depuis le début du $\mathrm{XX}^{\mathrm{e}}$ siècle sur la côte atlantique. Pour l'ensemble des régions de plaines de la planète, la vitesse moyenne de déplacement du climat pourrait dépasser $70 \mathrm{~km}$ par décennie au cours de la seconde moitié du XXI ${ }^{e}$ siècle dans le cas du scénario RCP8.5 (figure 6). En montagne, compte tenu des pentes, à réchauffement égal le déplacement du
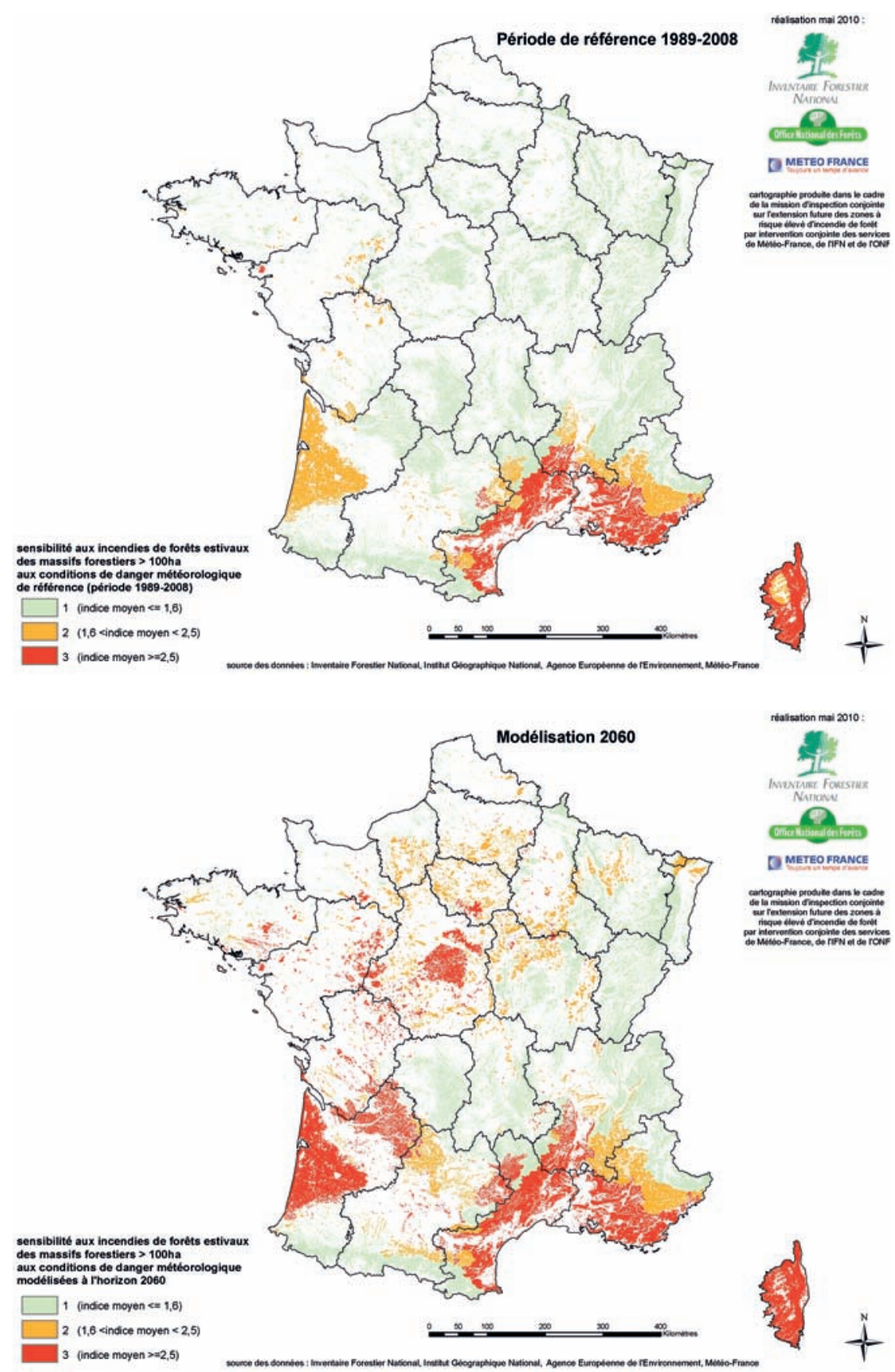

Figure 5. Cartographie des zones potentiellement sensibles aux incendies de forêt pour la période actuelle (à gauche) et pour I'horizon 2060 (à droite), sur la base de simulations réalisées avec le modèle Arpege-Climat de Météo-France. Le scénario futur retenu est le scénario d'émissions " médian » SRES-A1B.

climat correspondant est beaucoup plus faible qu'en plaine. Ainsi, toutes régions confondues, la vitesse moyenne de déplacement des climats est d'environ $20 \mathrm{~km}$ par décennie pour le scénario RCP8.5. La figure 6 (qui exclut les oiseaux) montre notamment que, dans le cas du scénario RCP2.6, toutes les espèces devraient être capables de migrer suffisamment rapidement et éviter l'extinction. Dans le cas du scénario RCP4.5, la migration des arbres serait trop lente en régions de plaine et beaucoup d'espèces pourraient être menacées sous le scénario RCP6.0 ou 8.5. Cependant, les interventions humaines, telles que par exemple le déplacement d'espèces ou la fragmentation des habitats, peuvent considérablement accélérer ou ralentir les mouvements migratoires.

Dans le cas de l'Europe, d'une manière générale, ce sont surtout les espèces résidant en altitude qui devraient être affectées par une dégradation de leur habitat. Les plantes actuellement présentes dans les montagnes européennes devraient migrer vers des altitudes plus élevées. Cela devrait conduire à un appauvrissement de la biodiversité dans les montagnes proches de la Méditerranée, mais à un enrichissement ailleurs. On estime par ailleurs que les zones de reproduction des oiseaux pourraient se décaler de près de $550 \mathrm{~km}$ vers le nord-est d'ici à la fin du $\mathrm{XXI}^{\mathrm{e}}$ siècle. La fragmentation des habitats aquatiques (rivières, zones humides, lacs) pourrait faire peser une menace supplémentaire sur la capacité de certaines espèces à se déplacer en réponse aux évolutions climatiques projetées. De même, l'augmentation de température risque d'induire une réduction des tailles des espèces animales en rendant les petits individus plus compétitifs pour l'exploitation des ressources, ce qui provoquera une réorganisation des écosystèmes, comme l'ont montré par exemple Edeline et al. (2013) dans le cas des poissons de rivière en France.

\section{Impact sur les écosystèmes marins}

Comme les écosystèmes terrestres et d'eau douce, les écosystèmes marins sont déjà affectés par le réchauffement de leur milieu. En particulier, les mers du nord de l'Europe (mer Baltique, mer du Nord) et la mer Noire se sont réchauffées 2 à 4 fois plus que la moyenne globale, ce qui a des effets 


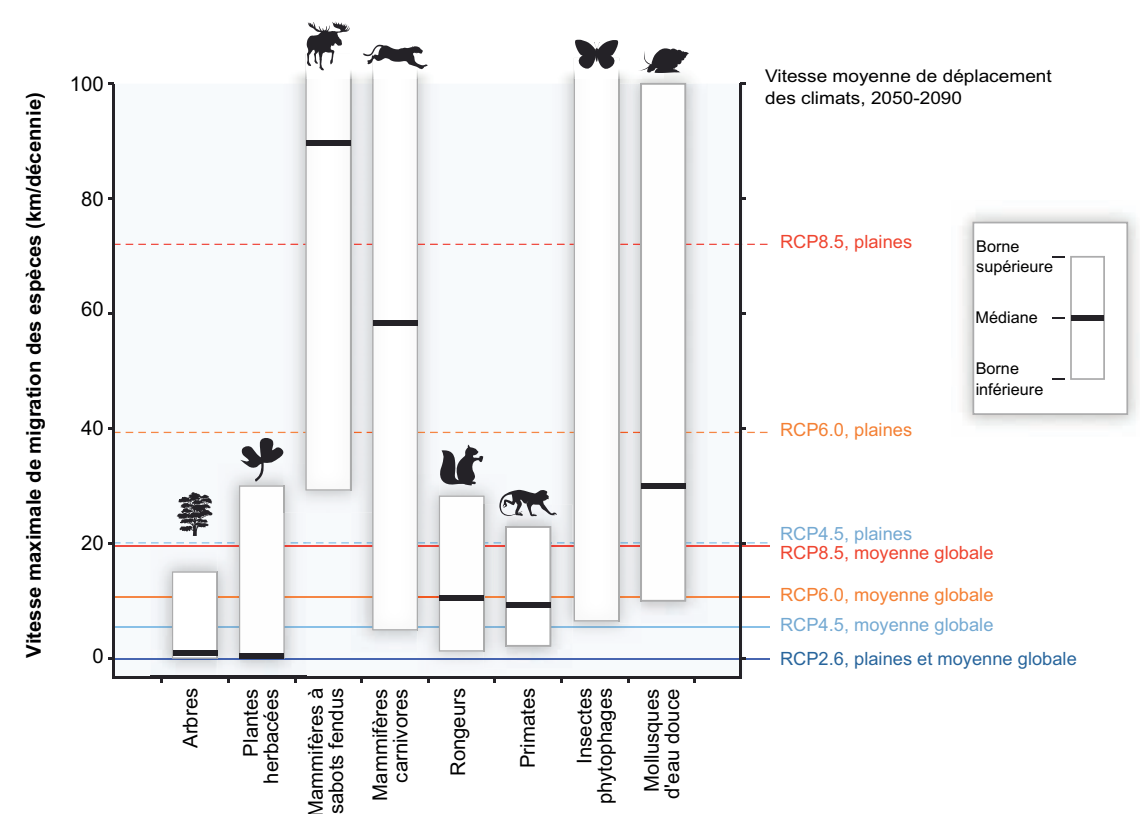

Figure 6. Comparaison de la vitesse maximale de migration des espèces (axe vertical de gauche) et de la vitesse d'évolution projetée des conditions de température (axe vertical de droite). Les rectangles blancs traversés d'un trait noir indiquent les fourchettes et les médianes des vitesses de déplacement maximales pour les arbres, les plantes, les mammifères, les insectes phytophages (médiane non estimée) et les mollusques d'eau douce. Les lignes horizontales colorées indiquent la vitesse moyenne de déplacement des climats sur les terres émergées du globe et la vitesse de ces déplacements sur les plaines et plateaux pour les scénarios RCP 2.6, 4.5, 6.0 et 8.5 pendant la période 20502090. Les espèces dont les vitesses maximales sont en dessous de chaque ligne devraient être incapables, en l'absence d'intervention humaine, de suivre le réchauffement correspondant à ce scénario. Source : Giec (2014), résumé à l'intention des décideurs.

négatifs sur les prises de poissons pour plusieurs raisons. Tout d'abord, d'une manière générale, ce réchauffement favorise les migrations de poissons vers le nord (Daufresne et al., 2009), sans remplacement systématique par d'autres espèces provenant du sud. Par exemple, il a été observé que les eaux de la Baltique deviennent trop chaudes pour certaines espèces actuellement présentes, mais la très faible salinité de cette mer ne la rend pas propice à la colonisation par d'autres poissons plus adaptés aux températures plus élevées. Par ailleurs, le réchauffement de l'eau de mer tend à réduire la taille des poissons marins (Daufresne et al., 2009). Enfin, dans certains cas, le réchauffement des eaux favorise le développement de certains parasites (Burge et al., 2014).

\section{Conséquence de l'acidification des océans}

Simultanément à d'autres changements globaux, tels que la diminution de la concentration d'oxygène dissous, et locaux (pollution et eutrophisation), les océans s'acidifient. Depuis le début de l'ère industrielle, les océans ont absorbé environ $30 \%$ des émissions humaines de $\mathrm{CO}_{2}$, entraînant une baisse du $\mathrm{pH}^{7}$ moyen de l'océan de surface de 0,1 unité. La pollution des eaux côtières, les remontées d'eaux profondes et les retombées d'azote et de soufre peuvent contribuer localement à l'acidification de l'eau de mer. Les processus chimiques sous-jacents à l'acidification sont très bien connus, mais il est plus difficile de prévoir l'acidification future des zones côtières en raison de leur complexité beaucoup plus grande que celle de l'océan hauturier (dynamique des masses d'eau et apports de matière organique et de sels nutritifs en provenance des continents).

L'acidification a des impacts positifs et négatifs, mais avec une grande marge d'incertitude car les organismes ont des niveaux de sensibilité très variables. Par ailleurs, les larves et les juvéniles ont fréquemment une sensibilité plus grande que les adultes. La diminution du $\mathrm{pH}$ provoque une diminution de la calcification de nombreux organismes calcaires vivant sur le fond, ce qui réduit leur compétitivité par rapport aux organismes non calcificateurs et augmente la dissolution des coquilles et squelettes calcaires. L'acidification stimule la croissance et la production primaire stimulées des plantes marines et de certaines espèces phytoplanctoniques. Les efflorescences d'algues toxiques pourraient devenir plus fréquentes et des perturbations comportementales ont été observées chez certains poissons. La biodiversité, la biomasse et la complexité du réseau trophique diminuent à proximité de sources sous-marines naturelles de $\mathrm{CO}_{2}$. Les conséquences de l'acidification combinées aux changements de la distribution des organismes provoqués par le réchauffement vont modifier les relations entre espèces, notamment les relations entre proies et prédateurs. Cela pourrait affecter les chaînes alimentaires, jusqu'à leurs niveaux supérieurs, comme les poissons d'intérêt commercial.

Quelques études montrent des capacités d'adaptation partielle à l'acidification de l'eau de mer, en particulier chez certaines algues planctoniques et chez certains mollusques. Cependant, les extinctions de masse survenues dans le passé durant des périodes d'acidification beaucoup plus lentes combinées avec d'autres modifications environnementales suggèrent que les mécanismes évolutifs sont trop lents pour que les espèces sensibles et à longue durée de vie puissent s'adapter aux changements à venir.

Les effets de l'acidification sur la biologie, l'écologie et la biogéochimie vont également affecter les services écosystémiques. En particulier, les océans vont devenir moins efficaces dans l'absorption du $\mathrm{CO}_{2}$ atmosphérique et donc dans la modération des changements climatiques. Les impacts de l'acidification, combinés à ceux du réchauffement (qui provoque le blanchissement des coraux) et à la montée du niveau de la mer, vont diminuer le rôle de protection des rivages des récifs coralliens. Cela ne manquerait pas d'entraîner des pertes de revenus pour l'industrie touristique et la pêche. Le coût global de la seule baisse de la production de mollusques pourrait s'élever à plus de 100 milliards de dollars d'ici 2100 (Narita et al., 2012). La propagation des effets sur la base de la chaîne alimentaire vers les niveaux supérieurs (poissons et mammifères marins) est très mal connue. Des modèles suggèrent que l'acidification va en général réduire la biomasse et la capture de poissons.

7. Le pH est une mesure de l'acidité utilisant une échelle logarithmique : une diminution du $\mathrm{pH}$ d'une unité correspond à une multiplication par 10 de la concentration des ions hydrogène. Le pH d'un jus de citron, de l'eau pure, de l'eau de mer et de l'eau de javel est d'environ 2,5, 7,0, 8,1 et 11,5 . 
(a) Réchauffement océanique, désoxygénation

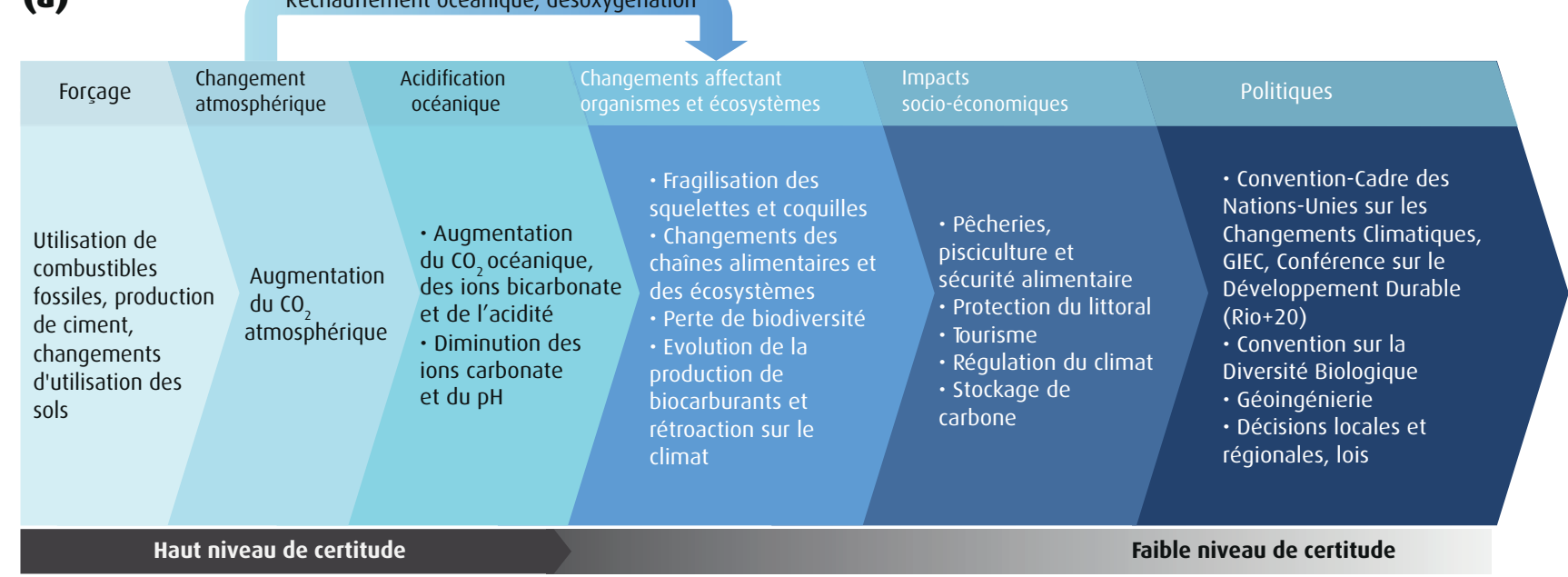

(b)

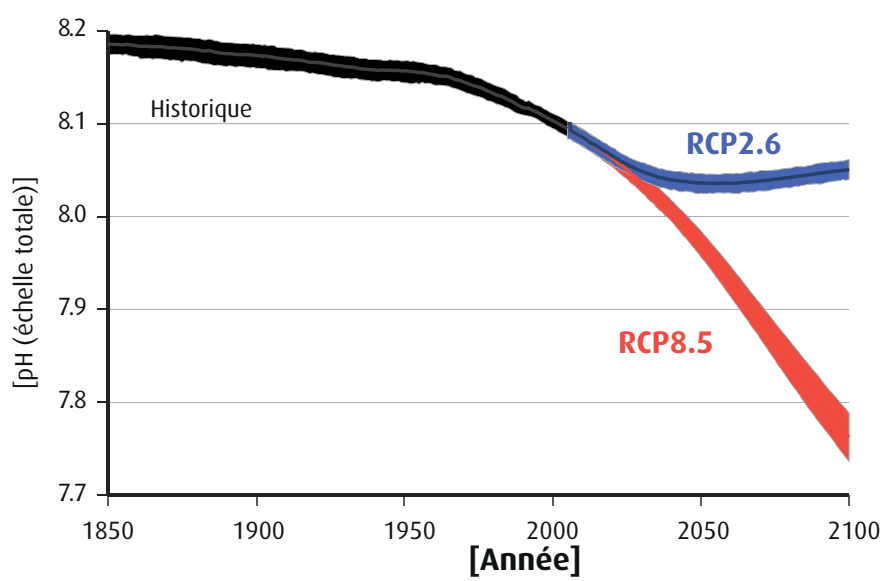

(c)

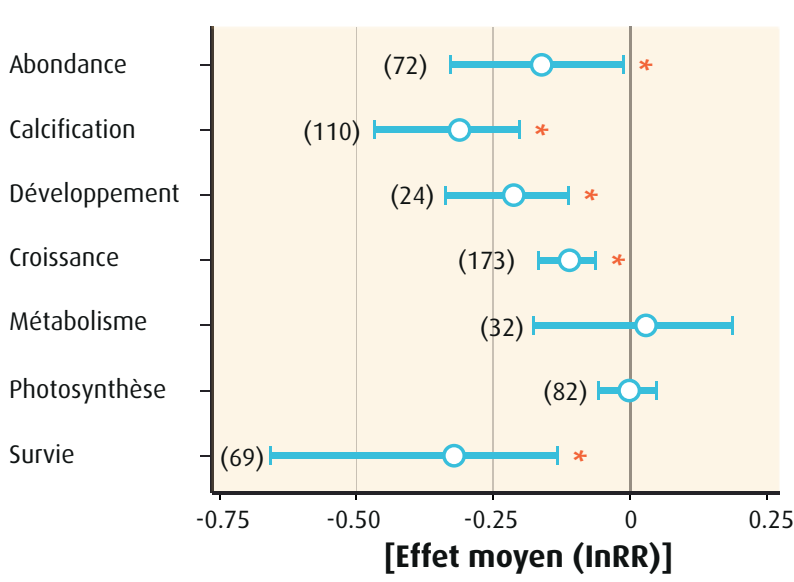

Figure 7. (a) Résumé des impacts chimiques, biologiques et socio-économiques liés à l'acidification des océans ainsi que ses implications sur la politique environnementale (adapté de Turley et Gattuso, 2012). (b) Série temporelle du pH (échelle totale) moyen des eaux de surface à l'échelle globale entre 1850 et 2100 à partir des simulations de modèles climatiques du projet CMIP5 (voir Boucher et al., 2015, ce numéro). Les projections sont fournies pour deux scénarios d'émissions : le RCP2.6 (bleu) et le RCP8.5 (rouge). Les moyennes multimodèles sont en traits pleins, tandis que les espaces compris entre les valeurs minimales et maximales sont colorés. La courbe en noir représente la modélisation de l'évolution historique. (c) Effets de l'acidification des océans (diminution de pH inférieure à 0,5 unité) sur les principales variables biologiques (Kroeker et al., 2013). Le nombre en abscisse représente une mesure de l'impact sur plusieurs processus (impact négatif si la valeur est négative, impact positif dans le cas inverse). La variabilité est assez grande entre espèces mais l'effet est statistiquement significatif lorsque les barres d'erreur n'incluent pas la valeur 0 (étoile rouge à droite dans ce cas). Le nombre d'expériences utilisées dans les analyses est indiqué entre parenthèses. Source : Giec (2014), résumé technique.

\section{Impacts et adaptation sur les secteurs des transports, de l'énergie, du tourisme}

\section{Transports}

Les connaissances relatives aux impacts directs du changement climatique sur les transports en Europe et plus encore à l'échelle mondiale sont encore relativement limitées. Le transport routier européen devrait être de moins en moins affecté par les événements neigeux et de verglas. Une augmentation du nombre d'accidents devrait prévaloir dans les régions où des précipitations futures augmentent, mais ces accidents devraient être moins graves, compte tenu de la tendance générale à la diminution des vitesses maximales autorisées. D’une manière générale, les accidents graves liés à des conditions météorologiques extrêmes devraient être moins fréquents, en raison de l'amélioration de la technologie des véhicules et des systèmes d'alerte. La multiplication des canicules projetée pour le $\mathrm{XXI}^{\mathrm{e}}$ siècle suggère que le transport ferroviaire pourrait être de plus en plus affecté par la déformation des rails (comme ce fut le cas en Grande-Bretagne lors de la canicule de 2003) ; en revanche, les dommages et retards liés à la neige et au gel devraient diminuer. Dans le cas du Rhin, on s'attend à ce que le niveau estival des eaux du fleuve soit suffisamment bas à partir de 2050 pour perturber significativement le transport fluvial. En effet, plus le niveau d'un fleuve est bas, moins les bateaux peuvent être chargés, ce qui augmente le coût de ce type de transport. Enfin, le retrait actuel et projeté de la glace de mer semble ouvrir de nouvelles possibilités de transport maritime intercontinental à travers l'océan Arctique, permettant de réduire les distances parcourues entre l'Europe et certaines destinations asiatiques. Cependant, ces opportunités doivent être relativisées en raison des dangers associés à la navigation en Arctique, du manque d'infrastructures, du coût des assurances et du peu d'intérêt actuel des 
armateurs pour inaugurer de nouvelles routes maritimes (Lasserre et Pelletier, 2011).

\section{Secteur de l'énergie}

Il n'est pas encore possible de détecter des impacts du changement climatique récent sur la production d'énergie. Pour le futur, le potentiel éolien ne devrait pas évoluer significativement avant 2050. Au-delà, il pourrait augmenter en hiver et diminuer en été en Europe centrale, en Europe du Nord et sur le flanc atlantique du continent. Dans le sud de l'Europe, une diminution en toutes saisons est attendue, sauf en mer Égée et sur la côte Adriatique, où le potentiel éolien pourrait significativement augmenter en été. En matière d'hydroélectricité, la situation est contrastée : en Scandinavie, la production pourrait augmenter de l'ordre de $10 \%$ d'ici à la fin du XXI ${ }^{\mathrm{e}}$ siècle, tandis qu'en Europe centrale et du Sud, et une partie des Alpes, elle pourrait diminuer d'environ $10 \%$ d'ici à 2050 . Il serait cependant possible de s'adapter à cette situation en améliorant la gestion de l'eau. Enfin, la production potentielle annuelle d'énergie solaire pourrait augmenter de 1'ordre de quelques pourcents entre 1990 et 2080 sur la plus grande partie de l'Europe (Crook et al., 2011).

\section{Tourisme}

Les évolutions climatiques futures devraient affecter les activités touristiques de manière très différente selon la région considérée. Après 2070, le climat d'été devrait être nettement plus favorable qu'aujourd'hui à la pratique d'activités touristiques "générales » dans une grande partie de l'Europe du Nord. En revanche, au-delà de 2050, les étés des régions méditerranéennes pourraient devenir trop chauds pour la pratique d'activités de plein air, tandis que les conditions devraient s'améliorer au printemps et en automne. Le tourisme d'été dans les régions de montagne pourrait être favorisé, mais la pratique des sports d'hiver devrait être plus aléatoire en raison d'un enneigement plus irrégulier dans certains massifs, et d'une diminution de la durée de la saison de ski. L'adaptation à un déficit d'enneigement consiste déjà à mettre en œuvre des canons à neige, mais le recours massif à ce type de technique est limité par les surcoûts économiques et par la disponibilité de la ressource en eau, en particulier dans les petites stations de faible altitude. Cependant, d'autres facteurs pourraient peser plus que l'évolution du climat sur la fréquentation des massifs montagneux en hiver, comme par exemple le vieillissement de la population.

\section{Santé}

Le changement climatique devrait avoir des impacts divers sur la santé des populations européennes. Pour la plupart des pays d'Europe, le plus grand danger lié à des températures extrêmes est représenté par les vagues de froid plutôt que par les canicules. Pour autant, la canicule d'août 2003 a entraîné une surmortalité de 15000 décès en France. Les canicules affectent principalement les populations sensibles telles que les personnes âgées ou les personnes souffrant d'une maladie chronique, résidant en particulier dans le sud de l'Europe, même si des risques peuvent être présents dans d'autres régions. Il existe relativement peu d'éléments sur l'efficacité de plans de prévention mis en œuvre dans plusieurs pays. Au cours du XXI e siècle, on s'attend à une augmentation des risques liés aux canicules et à une diminution de la mortalité liée aux vagues de froid. Les inondations liées à l'élévation du niveau des mers (Cazenave et al., 2015, ce numéro) et aux crues pourraient aussi entraîner des pertes en vies humaines difficilement quantifiables.

L'impact du changement climatique sur la propagation de certaines maladies à vecteurs en Europe doit être relativisé, les incertitudes étant encore grandes et les études relativement peu nombreuses. Le moustique tigre, vecteur de la dengue et du chikungunya, s'est récemment installé dans le sud de l'Europe et pourrait progresser vers l'est et le nord sous l'effet du changement climatique. La ré-émergence du paludisme en Europe est parfois évoquée, mais celle-ci ne serait sévère que dans le cas d'un afflux massif de personnes infectées ou de moustiques porteurs de la maladie, et d'une forte exposition des personnes aux moustiques. Dans ces deux cas, on estime que la propagation des maladies ne pourrait avoir lieu qu'à deux conditions : l'absence de contrôle efficace de la population de vecteurs et un affaiblissement des politiques sanitaires en Europe.

Enfin, une mauvaise qualité de l'air, c'est-à-dire de fortes concentrations de polluants (ozone, oxydes d'azote, particules fines diverses...) dans les basses couches de l'atmosphère, peut altérer la santé humaine en favorisant des maladies du système respiratoire, des maladies cardiovasculaires ou certaines formes de cancer. Au cours du $\mathrm{XXI}^{\mathrm{e}}$ siècle, en moyenne annuelle, l'évolution de la qualité de l'air devrait être principalement déterminée par les changements d'émissions de polluants liées aux activités humaines ou à la multiplication des incendies de forêts dans certaines régions. Ainsi, d'une manière générale, la qualité de l'air devrait s'améliorer dans la plupart des scénarios (sauf le RCP8.5), suite à l'application de réglementations dans différentes régions du monde. Par rapport au 4e rapport du Giec, la contribution du changement climatique à la qualité de l'air moyenne a été revue à la baisse, sauf en été dans certaines régions polluées (Kirtman et al., 2013). Plusieurs études suggèrent ainsi que la fréquence des pics de pollution à l'ozone pourrait augmenter dans certaines régions des États-Unis et d'Europe, lors d'épisodes météorologiques caractérisés par une " stagnation » des masses d'air. Cependant, au final, on s'attend à une diminution d'environ $5 \mathrm{ppb}^{8}$ de la concentration d'ozone dans les basses couches de l'atmosphère en 2100 par rapport aux niveaux actuels (environ $30 \mathrm{ppb}$ ) pour les scénarios à émissions moyennes RCP4.5 et RCP6.0, et à une hausse de 3 ppb pour le scénario RCP8.5 (Kirtman et al., 2013). Cette différence s'explique essentiellement par le fait que dans le scénario RCP8.5, les émissions de méthane, gaz précurseur de l'ozone troposphérique, sont plus importantes que dans les autres scénarios.

\section{Aspects sociaux}

Il existe relativement peu d'études portant sur les possibles impacts sociaux $\mathrm{du}$ changement climatique futur. On peut estimer cependant que les changements affectant l'agriculture ou l'industrie du tourisme pourraient avoir des conséquences sur divers bassins d'emplois. Les dommages liés à des catastrophes naturelles telles que les inondations ou les tempêtes sont déjà significatifs et impliquent des déplacements temporaires de populations touchées. D'autres déplacements pourraient s'effectuer de manière programmée, par exemple dans des régions où il

8. L'unité ppb (parties par milliard) correspond à une molécule du gaz considéré pour un milliard de molécules d'air sec. 
n'est pas possible de faire face à l'érosion côtière de long terme (Cazenave $e t$ al., 2015, ce numéro). L'impact du changement climatique et de la pollution atmosphérique sur les bâtiments de grande valeur tels que les monuments historiques est également un sujet de préoccupation. Les fortes chaleurs, le gel, la pluie, la cristallisation du sel présent dans l'air sur les matériaux

\section{Impact du changement climatique sur les villes et possibilités d'adaptation}

Par la modification du milieu naturel, en particulier l'artificialisation des surfaces, les villes modifient le climat local. Le phénomène le plus connu est l'îlot de chaleur urbain qui se traduit par des températures plus élevées en ville par rapport à la campagne environnante. Il est particulièrement marqué la nuit, car la chaleur emmagasinée dans les infrastructures urbaines durant la journée limite le refroidissement nocturne. Cette augmentation de plusieurs degrés de la température de l'air peut générer un stress thermique important pour les habitants et aggraver les impacts sanitaires lors de canicules. Ce fut le cas en 2003 : le faible refroidissement nocturne observé a eu un effet catastrophique à Paris où une surmortalité exceptionnelle a été relevée.

Les effets du changement climatique, superposés au climat urbain déjà existant, peuvent ainsi exacerber la vulnérabilité des villes (Lemonsu et al., 2013). Dans ce contexte, une réflexion sur les stratégies d'atténuation des effets urbains et d'adaptation au changement climatique doit d'ores et déjà être entreprise par les acteurs institutionnels et les aménageurs urbains pour planifier les villes de demain. Les longues échelles de temps appréhendées dans les études climatiques rendent l'exercice ardu, car la ville apparaît alors comme un système dynamique où interfèrent expansion urbaine, mutation architecturale, et évolution progressive des technologies et des modes de vie.

De récents projets de recherche ont proposé des méthodologies de modélisation systémique et interdisciplinaire de la ville (Masson et al., 2014b) permettant de quantifier les impacts du changement climatique sur Paris à l'horizon 2100. Différents scénarios d'adaptation de la ville, associant politique de planification urbaine, amélioration des technologies du bâti et usages énergétiques des habitants, sont évalués au travers d'indicateurs de confort thermique, de ressources en eau pour l'arrosage, de consommation d'énergie pour le chauffage et la climatisation, et de production décentralisée d'énergie.

D'ici la fin du siècle, un usage généralisé de la climatisation paraît incontournable pour faire face à l'amplification des occurrences de canicules (Lemonsu et al., 2014). Ce levier d'action entraîne néanmoins une surconsommation d'énergie en été et dégrade encore le confort extérieur du fait des rejets de chaleur des climatiseurs (de Munck et al., 2013). Des actions plus vertueuses et durables permettent de contrecarrer ces effets : une amélioration des performances énergétiques du parc immobilier et surtout une prise de conscience collective pour un usage raisonné de la climatisation. Quant aux stratégies de verdissement, elles sont performantes uniquement lorsque la végétation est arrosée, ce qui pose la question de la ressource en eau, en particulier en période de sécheresse. Lors d'événements aussi extrêmes, des actions d'urgence, comme les plans canicules, ont montré leur efficacité et restent indispensables; elles peuvent être associées à des mesures d'arrosage des chaussées dans les centres urbains qui permettent de rafraîchir efficacement l'air ambiant. Les mesures d'adaptation souvent préconisées pour faire face aux fortes chaleurs peuvent avoir des rétroactions inattendues à l'échelle annuelle et saisonnière qu'il est important d'évaluer. L'effet rafraîchissant de la végétation arborée peut ainsi accentuer les besoins en chauffage en hiver (de Munck, 2014). L'évaluation des scénarios d'adaptation à l'échelle pluriannuelle apporte des informations complémentaires pertinentes. On retiendra, en particulier que la production annuelle d'énergie solaire qu'aurait une ville comme Paris avec une stratégie volontariste d'installation de panneaux solaires (Masson et al., 2014a) est comparable à la consommation d'énergie cumulée liée au chauffage et à la climatisation, mais avec des temporalités différentes. De la même façon, pour certaines stratégies de verdissement, les volumes d'eau ruisselés sur les surfaces urbaines sont du même ordre de grandeur que les besoins en eau pour l'arrosage de la végétation. Ces résultats laissent entrevoir des solutions intéressantes de gestion durable de l'environnement urbain. Néanmoins, d'importants verrous technologiques de stockage et de redistribution de l'énergie ou de l'eau demeurent.

La recherche progresse à grand pas sur l'adaptation des villes au changement climatique bien que certaines questions restent encore ouvertes. L'enrichissement de l'approche systémique peut permettre d'améliorer l'évaluation multicritères des impacts et doit s'accompagner d'une évaluation de la faisabilité et des coûts de mise en œuvre des stratégies d'adaptation.

poreux tels que la pierre, ou encore l'acidité de certaines particules liées à la pollution atmosphérique affectent la plupart des bâtiments. Ces facteurs seront amenés à évoluer en lien avec les changements attendus du climat et de la qualité de l'air, et la préservation du patrimoine bâti devra se traduire par des mesures d'adaptation ciblées en fonction des régions et des matériaux. Les paysages (zones humides, vignobles, alpages, garrigues...) qui ont été forgés par des siècles d'intervention humaine constituent également un patrimoine et devraient également évoluer en fonction du climat. Dans le cas des vignobles par exemple, des mesures d'adaptation au changement climatique sont déjà mises en œuvre dans certaines régions d'Europe (mesures technologiques, contrôle de la production, utilisation d'autres variétés de vigne et, dans une certaine mesure, relocalisation de vignobles...). La plupart des mesures d'adaptation ont cependant leurs limites. Par exemple, il n'est pas certain qu'à l'avenir les consommateurs acceptent de payer un vin donné au même prix qu'actuellement s'il n'est plus produit au même endroit et avec la même variété de raisin. De plus, la réglementation actuelle de certains pays (AOC dans le cas de la France) définit de manière très stricte dans quelle région il est autorisé de planter chaque variété de vigne. Il a été ainsi proposé d'assouplir la notion de terroir, en la liant à un savoir-faire et une histoire plutôt qu'à une région particulière.

\section{Conclusion}

Les impacts du changement climatique en Europe sont multiples, souvent multifactoriels et déjà sensibles dans de nombreux domaines, plus particulièrement dans les régions méditerranéennes qui sont particulièrement exposées. Face aux impacts attendus, le niveau de développement de l'Europe offre de nombreuses perspectives d'adaptation, même si les moyens qui peuvent être mis en œuvre diffèrent entre les pays d'Europe de l'Est et les pays d'Europe de l'Ouest et du Nord.

Le groupe II du Giec a noté un doublement des publications scientifiques dans son domaine entre 2005 et 2010 , l'adaptation étant de plus en plus étudiée. Pour autant, la plupart des études sont encore spécifiques à un secteur donné. Il faut s'attendre dans les années à venir à voir se développer des études 
multisectorielles, qui seront très utiles pour construire des politiques d'adaptation solides, ainsi que des études visant à évaluer scientifiquement les politiques d'adaptation menées à tous les niveaux.

Les mesures d'adaptation sont un levier d'action pour limiter les impacts du changement climatique, il est donc essentiel de choisir les bonnes stratégies puis de les appliquer. L'autre levier d'action consiste à directement atténuer le réchauffement climatique par la réduction des émissions de gaz à effet de serre au plan mondial (Guivarch et Cassen, 2015, ce numéro).

\section{Remerciements}

Les auteurs remercient les relecteurs pour leurs commentaires très utiles pour la finalisation de cet article.

\section{Bibliographie}

Acta-Mires, 2009. Recueil de communications du colloque " Changement climatique : conséquences et enseignements pour les grandes cultures et l'élevage herbivore ", Arvalis, Institut de l'élevage, Inra, Météo-France, Paris, 22 octobre 2009, Programme Acta-Mires "Changements climatiques ».

AEE, 2012. Climate change, impacts and vulnerability in Europe. An indicator-based report, 2012. EEA report №12/2012, Agence européenne de l'environnement, Copenhague. doi: 10.2800/66071

Boucher 0., Dufresne J.-L., Vial J., Brun E., Cattiaux J., Chauvin F., Salas y Mélia D., Voldoire A., Bopp L., Braconnot P., Ciais P., Yiou P., Guilyardi E., Mignot J., Guivarch C., 2015. Projection des changements climatiques futurs. La Météorologie, 88, 56-68.

Brisson N., Gate P., Gouache D., Charmet G., Oury F.-X., Huard F., 2010. Why are wheat yields stagnating in Europe? A comprehensive data analysis for France. Field Crops Research, 119, 201-212.

Burge C.A., Eakin C.M., Friedman C.S., Froelich B., Hershberger P.K., Hofmann E.E., Petes L.E., Prager K.C., Weil E., Willis B.L., Ford S.E., Harvell C.D., 2014. Climate change influences on marine infectious diseases: implications for management and society. Annu. Rev. Mar. Sci., 6, 249-277.

Cazenave A., Berthier E, Meyssignac B., Le Cozannet G., Masson-Delmotte V., Salas y Mélia D., 2015. Le niveau de la mer : variations passées, présentes et futures. La Météorologie, 88, 69-82.

Chauveau M., Chazot S., Perrin C., Bourgin P.-Y., Sauquet E., Vidal J.-P., Rouchy N., Martin E., David J., Norotte T., Maugis P., De Lacaze X., 2013. Quels impacts des changements climatiques sur les eaux de surface en France à l'horizon 2070 ? La Houille Blanche, 4, 5-15.

Cheaib A., François C., Badeau V., Boe J., Chuine I., Delire C., Dufrêne E., Gritti E., Legay M., Page C., Thuillier W., Viovy N., Leadley P., 2012. Climate change impacts on tree ranges: model inter-comparison facilitates understanding and quantification of uncertainty. Ecol. Lett., 15 (6), 533-544. doi: 10.1111/j.1461-0248.2012.01764.x

Crook J.A., Jones L.A., Forster P.M., and Crook R., 2011. Climate change impacts on future photovoltaic and concentrated solar power energy output. Energy Environ. Sci., 4(9), 3101-3109.

Daufresne M., Lengfellner K., Sommer U., 2009. Global warming benefits the small in aquatic ecosystems. Proceedings of the National Academy of Sciences of the United States of America, 106(31), 12788-93.

De Munck C., Pigeon G., Masson V., Meunier F., Bousquet P., Tréméac B., Merchat M., Poeuf P., Marchadier C., 2013. How much air conditioning can increase air temperatures for a city like Paris (France)? Int. J. Climatol., 33, 210-227. doi: 10.1002/joc.3415

De Munck C., 2014. Modélisation de la végétation urbaine et stratégies d'adaptation pour l'amélioration du confort climatique et de la demande énergétique en ville. Thèse Université de Toulouse, Toulouse, $219 p$.

Edeline E., Lacroix G., Delire C., Poulet N., Legendre S., 2013. Ecological emergence of thermal clines in body size. Global Change Biol., 19, $3062-3068$.

Giec, 2014. Climate Change 2014 : Impacts, Adaptation and Vulnerability. Part A : Global and sectoral aspects. Contribution of Working group II to the Fifth Assessment Report of the Intergovernmental Panel on Climate Change. Field C.B., Barros V.R., Dokken D.J., Mach K.J., Mastrandrea M.D., Bilir T.E., Chatterjee M., Ebi K.L, Estrada Y.O., Genova R.C., Girma B., Kissel E.S., Levy A.N., MacCracken S., Mastrandrea P.R. et White L.L. Eds. Cambridge University Press, Cambridge, UK, New York, NY, USA ,1132 p.

Guivarch C., Cassen C., 2015. L'atténuation du changement climatique: retour sur le 5e rapport du Giec. La Météorologie, 88, 97-105.

Hallegatte S., 2009. Strategies to adapt to an uncertain climate change. Global Env. Change, 19, 240-247.

Kovats R.S., Valentini R., Bouwer L.M., Georgopoulou E., Jacob D., Martin E., Rounsevell M. and Soussana J.-F., 2014. Europe, In: Field C.B., Barros V.R., Dokken D.J., Mach K. J., Mastrandrea M. D., Bilir T.E., Chatterjee M., Ebi K.L., Estrada Y.O., Genova R.C., Girma B., Kissel E.S., Levy A.N., MacCracken S., Mastrandrea P.R., White L.L., eds. Climate Change 2014: Impacts, Adaptation, and Vulnerability. Part B: Regional Aspects. Contribution of Working Group II to the Fifth Assessment Report of the Intergovernmental Panel on Climate Change. Cambridge University Press, Cambridge, UK, New York, NY, USA, 1267-1326.

Kirtman B., Power S.B., Adedoyin A.J., Boer G.J., Bojariu R., Camilloni I., Doblas-Reyes F., Fiore A.M., Kimoto M., Meehl G., Prather M., Sarr A., Schär C., Sutton R., van Oldenborgh G.J., Vecchi G., Wang H.-J., 2013. Near-term Climate Change: Projections and Predictability, In Stocker T.F., Oin D., Plattner G.-K., Tignor M., Allen S.K., Boschung J., Nauels A., Xia Y., Bex V., Midgley P.M., Eds. Climate Change 2013: The Physical Science Basis. Contribution of Working Group I to the Fifth Assessment Report of the Intergovernmental Panel on Climate Change. Cambridge University Press, Cambridge, UK, New York, NY, USA, 953-1028.

Kroeker K., Kordas R.C., Ryan A., Hendriks I., Ramajo L., Singh G., Duarte C., Gattuso J.-P., 2013. Impacts of ocean acidification on marine organisms: quantifying sensitivities and interaction with warming. Global Change Biol., 19, 1884-1896.

Lasserre, F., Pelletier S., 2011. Polar super seaways? Maritime transport in the Arctic: an analysis of shipowners' intentions. J. Transp. Geogr., 19, $1465-1473$.

Lemonsu A., Kounkou-Arnaud R., Desplat J., Salagnac J.-L., Masson V., 2013. Evolution of the Parisian urban climate under a global changing climate. Clim. Change, 116 , 679-692.

Lemonsu A., Beaulant A.-L., Somot S., Masson V., 2014. Evolution of heat wave occurrence over the Paris basin (France) in the 21st century. Clim. Res., 61, 75-91.

Masson V., Bonhomme M., Salagnac J.-L., Briottet X., Lemonsu A., 2014a. Solar panels reduce both global warming and urban heat island. Front. Environ. Sci., 2 : 14. doi: 10.3389/fenvs.2014.00014

Masson V., Marchadier C., Adolphe L., Aguejdad R., Avner P., Bonhomme M., Bretagne G., Briottet X., Bueno B., de Munck C., Doukari O., Hallegatte S., Hidalgo J., Houet T., Le Bras J., Lemonsu A., Long N., Moine M.-P., Morel T., Nolorgues L., Pigeon G., Salagnac J.-L., Zibouche K., 2014b. Adapting cities to climate change: a systemic modelling approach. Urban Climate, 10, 407-429. doi: 10.1016/j.uclim.2014.03.004 
Masson-Delmotte V., Braconnot P., Kageyama M., Sépulchre, P., 2015. Qu'apprend-on des grands changements climatiques passés ? La Météorologie, 88, 25-35. Narita D., Rehdanz K., Tol R.S.J., 2012. Economic costs of ocean acidification: a look into the impacts on shellfish production. Clim. Change, 113, 1049-1063.

Planton P., Bopp L., Brun E., Cattiaux J., Chauvin F., Chevallier M., Ciais P., Douville H., Giraud G., Soubeyroux J.-M., Terray L., 2015. Le climat depuis 1850. La Météorologie, 88, 48-55

Porter J.R., Xie L., Challinor A.J., Cochrane K., Howden S.M., Mohsin Iqbal M., Lobell D.B., Travasso M.I., 2014. Food security and food production systems. In: Field C.B., Barros V.R., Dokken D.J., Mach K. J., Mastrandrea M. D., Bilir T.E., Chatterjee M., Ebi K.L., Estrada Y.O., Genova R.C., Girma B., Kissel E.S., Levy A.N., MacCracken S. Mastrandrea P.R., White L.L., eds. Climate Change 2014: Impacts, Adaptation, and Vulnerability. Part A: Global and Sectorial Aspects. Contribution of Working Group II to the Fifth Assessment Report of the Intergovernmental Panel on Climate Change. Cambridge University Press, Cambridge, UK, New York, NY, USA, 485-533.

Turley C., Gattuso J.-P., 2012. Future biological and ecosystem impacts of ocean acidification and their socioeconomic-policy implications. Curr. Opin. Environ. Sustain., 4, 278-286.

Vert J., Schaller N., Villien C., 2013. Agriculture, forêt, climat : vers des stratégies d'adaptation. Centre d'études et de prospective, ministère de l'Agriculture, de l'Agroalimentaire et de la Forêt, 234 p. 
L'Agence De l'Environnement et de la Maîtrise de l'Énergie (ADEME) accompagne les collectivités territoriales dans leurs démarches d'adaptation au changement climatique.

Cet accompagnement prend la forme de recueils d'expérience, de guides méthodologiques et de formations.

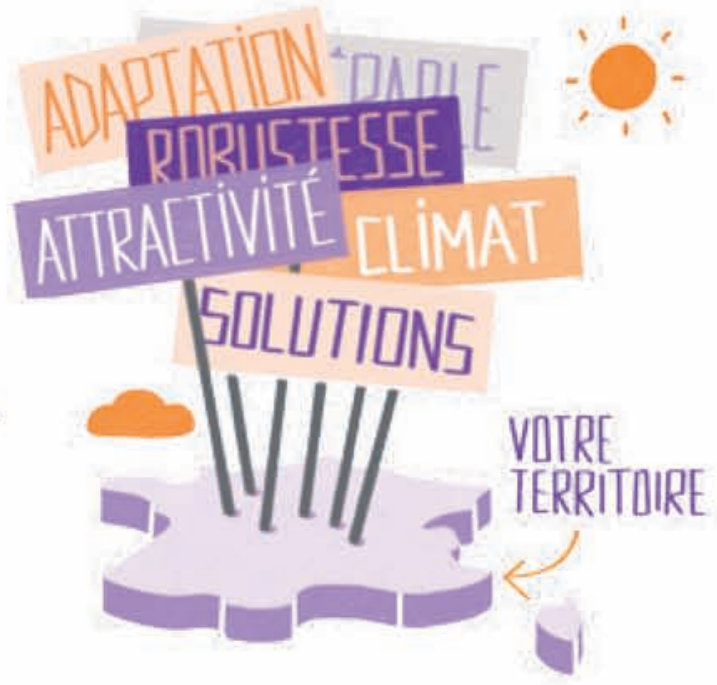

\section{Impact Climat}

\section{Objectif' Climat}

\section{LA DÉMARCHE IMPACT'CLIMAT}

permet aux collectivités territoriales d'entamer leur démarche d'adaptation au changement climatique, à travers la réalisation d'un diagnostic de limpact du changement climatique sur leur territoire.

La démarche propose une approche pragmatique basée sur l'exploitation des connaissances déjà disponibles sur le changement climatique et ses impacts. Un guide présente l'approche méthodologique, liste les ressources existantes et accompagne l'utilisateur lors des différentes étapes de l'analyse. Un tableur Excel propose des grilles d'analyse et des présentations graphiques d'indicateurs clés, dont les 19 indices climatiques de référence pour la France.

\section{LA DÉMARCHE OBJECTIF'CLIMAT}

permet aux collectivités territoriales de structurer leur politique d'adaptation au changement climatique et le dispositif de suivi-évaluation de celle-ci. Elle propose une méthodologie permettant d'évaluer sa progression et ses performances, dans une logique d'amélioration continue et d'apprentissage collectif.
Le système de suivi-évaluation proposé repose sur le « cadre logique », éprouvée dans de nombreuses politiques et projets de territoire (Agenda2I, etc.). Des questions évaluatives spécifiques à la problématique de l'adaptation au changement climatique sont proposées pour la phase d'évaluation finale.

\section{FORMATIONS PLANS CLIMAT-ENERGIETERRITORIAUX}

L'ADEME a élaboré plusieurs modules de formation dédiés aux Plans Climat-Energie Territoriaux. Un module de deux jours traite spécifiquement du volet « adaptation au changement climatique » dans les politiques locales et forme les chargés de mission PCET aux méthodes de diagnostic, d'élaboration de stratégies et plans d'actions ainsi qu'au suivi et à l'évaluation des politiques d'adaptation.

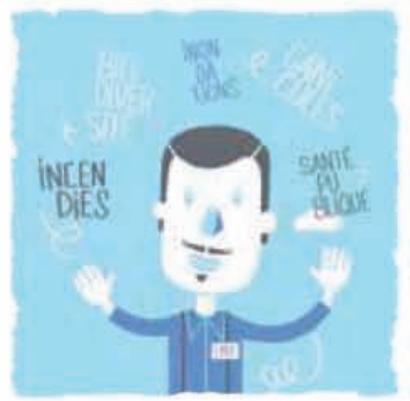

\section{L'ADEME EN BREF}

L'Agence de l'Environnement et de la Maîtrise de l'Energie (ADEME) participe à la mise en œuvre des politiques publiques dans les domaines de l'environnement, de l'énergie et du développement durable. Elle met ses capacités d'expertise et de conseil à disposition des entreprises, des collectivités locales, des pouvoirs publics et du grand public, afin de leur permettre de progresser dans leur démarche environnementale. L'Agence aide en outre au financement de projets, de la recherche à la mise en œuvre et ce, dans les domaines suivants : la gestion des déchets, la préservation des sols, l'efficacité énergétique et les énergies renouvelables, la qualité de l'air et la lutte contre le bruit.

L'ADEME est un établissement public sous la tutelle conjointe du ministère de l'Ecologie, du Développement durable et de l'Energie et du ministère de l'Education nationale, de l'Enseignement supérieur et de la Recherche.

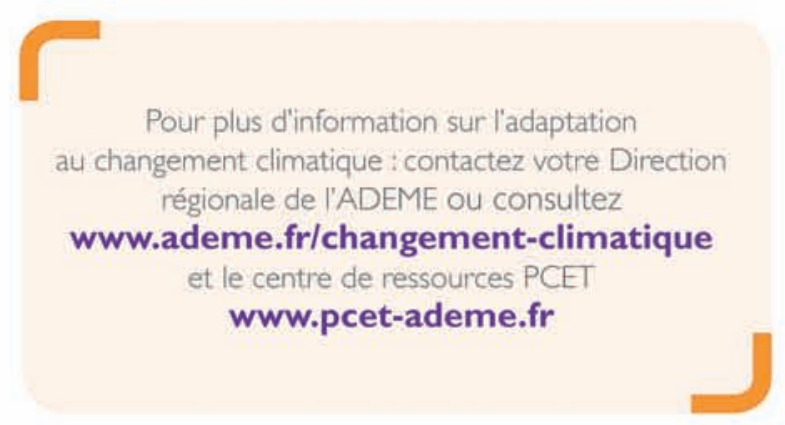

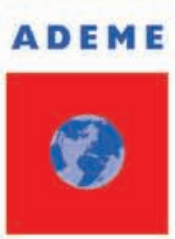

Agence de l'Environnement et de la Maîtrise de l'Energie 